\title{
Accuracy assessment of noninvasive cardiac output monitoring in the hemodynamic monitoring in critically ill patients
}

\author{
Guijun Zhu, Kun Zhang, Yuxin Fu, Zhenjie $\mathrm{Hu}^{\wedge}$ \\ Department of Critical Care Medicine, The Fourth Hospital of Hebei Medical University, Shijiazhuang, China \\ Contributions: (I) Conception and design: Z Hu, G Zhu, K Zhang; (II) Administrative support: None; (III) Provision of study materials or patients: F \\ Xin; (IV) Collection and assembly of data: F Xin; (V) Data analysis and interpretation: K Zhang, F Xin; (VI) Manuscript writing: All authors; (VII) \\ Final approval of manuscript: All authors. \\ Correspondence to: Zhenjie Hu. Department of Critical Care Medicine, The Fourth Hospital of Hebei Medical University, Shijiazhuang, China. \\ Email: syicu@vip.sina.com.
}

Background: The consistency of cardiac output (CO) measured by noninvasive cardiac output monitoring (NICOM), pulse index continuous cardiac output (PiCCO), and ultrasound in the hemodynamic monitoring of critically ill patients was studied. Using the NICOM built-in passive leg raising (PLR) test, stroke volume index variation $(\Delta \mathrm{SVI})$ was calculated and was used to predict volume responsiveness in patients with circulatory shock (excluding cardiogenic shock).

Methods: Critically ill patients requiring hemodynamic monitoring were admitted during the study period. The CO of each included patient under hemodynamic monitoring was measured by NICOM plus PiCCO or ultrasound, and the consistency of the measured COs was analyzed. By the NICOM built-in PLR test, $\Delta$ SVI was calculated and was used to predict volume responsiveness.

Results: The CO of 58 patients was measured by NICOM and ultrasound, and the COs measured by these two methods were consistent. The CO of 40 patients was measured by NICOM and PiCCO, and the COs measured by these two methods were consistent. The volume responsiveness of all 98 patients was assessed by the NICOM built-in PLR test. A total of 60 patients had $\triangle$ SVI $>10 \%$, so they underwent the fluid challenge. Among them, 43 patients were positive by both the NICOM built-in PLR and fluid challenge. When using $\triangle \mathrm{SVI}$ to predict volume responsiveness in patients with circulatory shock (excluding cardiogenic shock), the area under the receiver operating characteristic curve was 0.754 (95\% confidence interval, 0.6260.856 ), and the cut-off value was $18 \%$ (sensitivity: $88.37 \%$, specificity: $52.94 \%$ ), indicating that $\Delta$ SVI has value in predicting the volume responsiveness of patients with noncardiogenic circulatory shock.

Conclusions: NICOM had good consistency with ultrasound and PiCCO in the hemodynamic monitoring of critically ill patients and can be for hemodynamic monitoring and evaluation in critically ill patients. The $\triangle$ SVI obtained by the NICOM built-in PLR test has certain clinical value in predicting the volume responsiveness of patients with circulatory shock (excluding cardiac shock) and provides a method for evaluating the volume responsiveness of critically ill patients.

Keywords: Noninvasive cardiac output monitor (NICOM); cardiac output (CO); volume responsiveness; passive leg raising test; stroke volume index

Submitted Jul 24, 2020. Accepted for publication Sep 14, 2020.

doi: 10.21037/apm-20-1731

View this article at: http://dx.doi.org/10.21037/apm-20-1731

$\wedge$ ORCID: 0000-0003-1404-5691. 


\section{Introduction}

The best treatment for critically ill patients depends on the accurate assessment of their hemodynamic status. Perel et al. showed that in the absence of advanced hemodynamic monitoring, clinicians are limited to predicting important physiological indicators only from their clinical examination, but the use of advanced hemodynamic monitoring in critically ill patients could help in this prediction (1). Hemodynamic monitoring can reliably determine the type of shock, help clinicians choose the most suitable treatment, and evaluate the treatment efficacy (2).

Advanced hemodynamic monitoring is the basis for the treatment of critically ill patients. Over the past several decades, hemodynamic monitoring techniques have evolved from intermittent monitoring to continuous, real-time monitoring and from invasive monitoring to noninvasive monitoring. The hemodynamic parameters provided are also more comprehensive. The accuracy of SwanGanz catheter and pulse index continuous cardiac output (PiCCO) as a method of measuring cardiac output (CO) has been recognized, but it is an invasive operation (3); critical ultrasound can As a simple and fast non-invasive hemodynamic monitoring technology, the accuracy of the monitoring data may be closely related to the operator's proficiency or operating specifications (4); Noninvasive cardiac output monitor (NICOM) can perform noninvasive, continuous and relatively accurate hemodynamics Scientific monitoring is gradually applied to the clinic. A series of studies have shown that NICOM has a good correlation with thermodilution (5) and PiCCO (6), and can replace traditional invasive or minimally invasive hemodynamic monitoring techniques. In predicting volume responsiveness of critically ill patients, the stroke volume index variation $(\triangle S \mathrm{SVI})$ measured by NICOM can successfully predict fluid responsiveness during prone surgery (7).

This study aimed to evaluate the consistency of cardiac output (CO) measured by NICOM, pulse index continuous cardiac output (PiCCO), and ultrasound in the hemodynamic monitoring of critically ill patients and to use the stroke volume index variation $(\Delta \mathrm{SVI})$ calculated by the NICOM built-in passive leg raising (PLR) test to predict volume responsiveness in patients with circulatory shock (excluding cardiogenic shock). The authors have completed the STARD reporting checklist (available at http://dx.doi. org/10.21037/apm-20-1731).

\section{Methods}

\section{Patient selection}

The critically ill patients requiring hemodynamic monitoring in the Department of Critical Care Medicine, The Fourth Hospital of Hebei Medical University, from December 2018 to January 2020 were included.

\section{Inclusion criteria}

(I) Systolic blood pressure below $90 \mathrm{mmHg}$, or $40 \mathrm{mmHg}$ lower than the original baseline value, or the need for vasoactive drugs to maintain blood pressure; (II) insufficient tissue perfusion and lactic acid greater than $2.0 \mathrm{mmol} / \mathrm{L}$, or clammy skin, or urine output less than $0.5 \mathrm{~kg} / \mathrm{ml} / \mathrm{h}$, or acute disturbance of consciousness; (III) fatal organ dysfunction due to host response to severe infection, i.e., acute change in Sequential Organ Failure Assessment (SOFA) score caused by infection $\geq 2$ points.

\section{Exclusion criteria (part 1)}

(I) Insufficient skin where the NICOM sensors are placed; (II) contraindications for femoral artery catheterization; (III) unclear cardiac findings on ultrasound examination; (IV) severe pulmonary arterial hypertension (pulmonary arterial pressure greater than $60 \mathrm{mmHg}$ ); (V) severe aortic insufficiency and tricuspid insufficiency; (VI) age $<18$ years or pregnancy; (VII) inability to perform the PLR test; (VIII) for the fluid challenge, acute coronary syndrome, cardiogenic shock, or significant volume overload; (IX) refusal to sign the informed consent form.

\section{Exclusion criteria (part 2)}

(I) Patients with incomplete data collection; (II) patients who met the inclusion criteria and withdrew from the study.

\section{Ethics}

This study followed medical ethics standards and was approved by the medical ethics committee of the hospital (approval number: 2019117). All procedures performed in this study involving human participants were in accordance with the Declaration of Hels inki (as revised in 2013).The patients or their family members were informed about the study protocols and signed informed consent forms.

\section{Methodology}

(I) $\mathrm{CO}$ monitored by NICOM: four dual-electrode stickers 
were attached to the patient's chest around the heart. The electrode was connected to the NICOM device through four wires. After the NICOM was automatically calibrated for 90 seconds, NICOM continuously monitored CO, and $\mathrm{CO}$ was recorded. (II) $\mathrm{CO}$ monitored by PiCCO: advanced hemodynamics monitoring by $\mathrm{PiCCO}$ was performed according to the operating specifications. The average $\mathrm{CO}$ of three measurements was taken for analysis. (III) $\mathrm{CO}$ monitored by ultrasound: each patient was examined by a bedside echocardiograph by a physician who had undergone rigorous ultrasound training. The velocitytime integral (VTI) of aortic blood flow was calculated using pulsed Doppler echocardiography, and the average VTI of three measurements was taken. (IV) Volume responsiveness predicted by the NICOM built-in PLR test: the NICOM built-in PLR test was used. The patient was in the semirecumbent position, with the legs flat on the bed, and the baseline level was measured for 3 minutes. After the patient's upper body was laid flat, the legs were raised to 30-45 degrees with a standard wedge pillow, and the challenge level was measured for 3 minutes. If the $\Delta$ SVI exceeded $10 \%$, the clinicians evaluated the condition of the patient first, then the 'classic' fluid challenge was performed, and the change rate of $\mathrm{CO}$ before and after fluid infusion was recorded. (V) Management of emergencies during the study: if the patient breathed heavily or had increased moist rhonchi in the lungs during the fluid resuscitation, the fluid resuscitation was terminated immediately. During PLR and fluid resuscitation, the types and doses of drugs were kept unchanged, and ventilator parameters were not adjusted.

The following indicators were recorded: (I) general information of the patients: sex, age, height, and body weight; (II) main reason for intensive care unit (ICU) admission: cardiopulmonary resuscitation, trauma, acute pancreatitis, or surgery; (III) heart rate (HR), mean arterial pressure (MAP), lactate level (Lac), CO, and $\triangle$ SVI.

\section{Statistical methods}

SPSS version 22.00 was used for statistical analysis. Measurement data with a normal distribution are expressed as the mean \pm standard deviation. Bland-Altman analysis was used to quantify the consistency between different $\mathrm{CO}$ measurements to evaluate the reliability of our NICOM monitoring results. Using MedCalc19.1.3, the predictive value of $\triangle S V I$ was statistically analyzed through the receiver operating characteristic (ROC) curve. The area under the curve (AUC) was used to represent the evaluation results, and its cut-off value, sensitivity, and specificity were calculated. $\mathrm{P}<0.05$ was considered statistically significant.

\section{Results}

\section{Basic medical characteristics of the included patients}

A total of 682 patients were admitted to our department during the study period. One hundred patients were included in strict accordance with the inclusion and exclusion criteria. Two patients were excluded due to incomplete data, so 98 patients were included in this study. The 98 included patients were statistically analyzed (Table 1).

\section{Consistency analysis of $\mathrm{CO}$ monitored by different methods}

\section{Consistency of CO between NICOM and ultrasound (Figure 1)}

The consistency of CO between NICOM and ultrasound was investigated in 58 patients. There was a bias of $0.42 \mathrm{~L} / \mathrm{min}$ between the two methods. Figure 1 shows that five out of 58 points fell outside the $95 \%$ confidence interval (CI), a percentage of $8.6 \%$ (less than $10 \%$ ). Within the CI, the maximum difference (absolute value) was $1.89 \mathrm{~L} / \mathrm{min}$. With a maximum difference between the two measurements of $1.89 \mathrm{~L} / \mathrm{min}$ and an average of $3.56 \mathrm{~L} / \mathrm{min}$ for both methods show, this difference is clinically acceptable, so both methods can be used interchangeably.

\section{Consistency of CO between NICOM and PiCCO (Figure 2)}

The consistency of CO by NICOM and PiCCO was investigated in 40 patients. There was a bias of $0.51 \mathrm{~L} / \mathrm{min}$ between the two methods, and the $95 \% \mathrm{CI}$ interval was -0.75 to $1.76 \mathrm{~L} / \mathrm{min}$. Figure 2 shows that three out of the 40 points fell outside the $95 \% \mathrm{CI}$, a percentage is $7.5 \%$ (less than 10\%). Within the CI, the maximum difference (absolute value) was $1.62 \mathrm{~L} / \mathrm{min}$. With a maximum difference between the two measurements of $1.62 \mathrm{~L} / \mathrm{min}$ and an average of $5.99 \mathrm{~L} / \mathrm{min}$ for both methods, this difference is clinically acceptable, so both methods can be used interchangeably.

\section{Evaluation of the predictive value of $\Delta S V I$ for the volume responsiveness in patients with circulatory shock (noncardiogenic) (Figure 3)}

The volume responsiveness in all 98 patients was assessed by the NICOM built-in PLR test. Among them, 38 had 


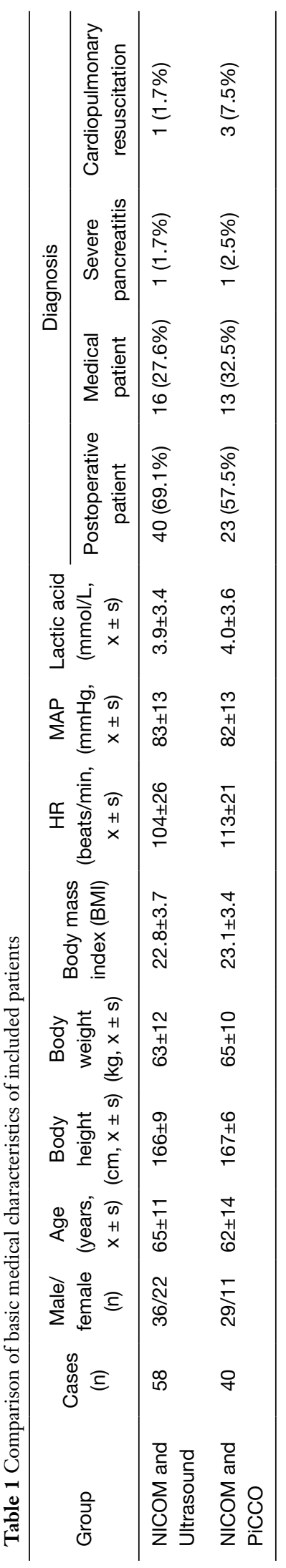

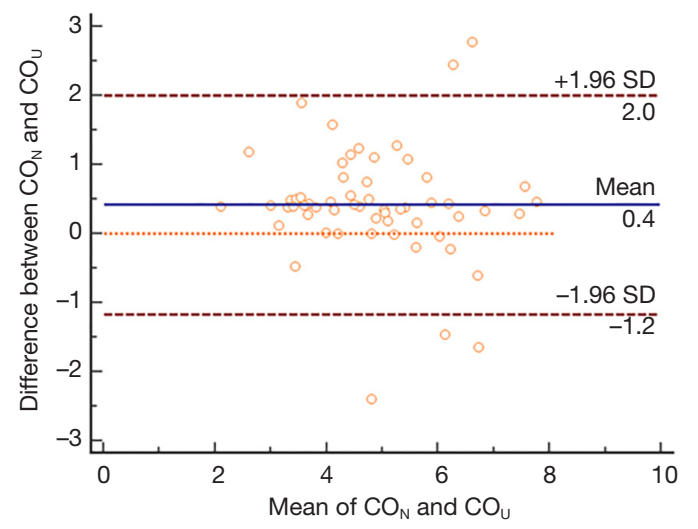

Figure 1 Bland-Altman plot of $\mathrm{CO}_{\mathrm{N}}$ and $\mathrm{CO}_{\mathrm{U}} \cdot \mathrm{CO}_{\mathrm{N}}, \mathrm{CO}$ monitored by NICOM; $\mathrm{CO}_{\mathrm{U}}$, $\mathrm{CO}$ monitored by ultrasound.

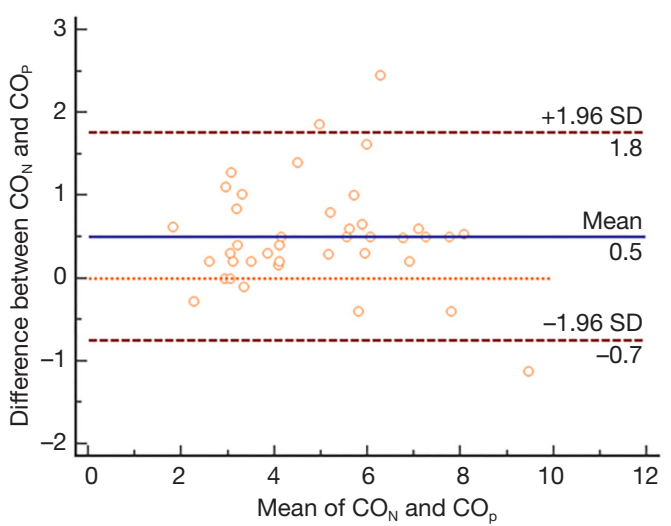

Figure 2 Bland-Altman plot of $\mathrm{CO}_{\mathrm{N}}$ and $\mathrm{CO}_{\mathrm{p}} \cdot \mathrm{CO}_{\mathrm{N}}$, $\mathrm{CO}$ monitored by NICOM; $\mathrm{CO}_{\mathrm{P}}$, $\mathrm{CO}$ monitored by PiCCO.

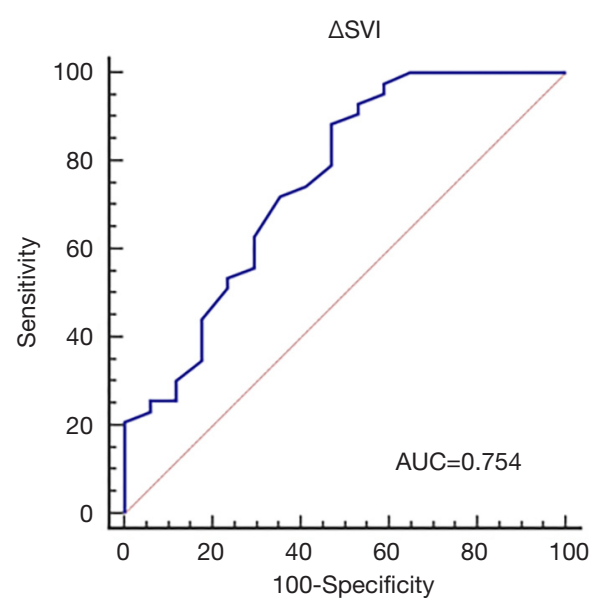

Figure 3 Evaluation of volume responsiveness predicted by $\Delta$ SVI in patients with circulatory shock (noncardiogenic). $\Delta \mathrm{SVI}$, stroke volume index variation; $\mathrm{AUC}$, area under the ROC curve. 
$\Delta$ SVI $<10 \%$, and after the comprehensive evaluation of their condition, they did not undergo the fluid challenge. The $\Delta$ SVI of the other 60 patients was greater than $10 \%$, suggesting that there might be volume responsiveness. After the comprehensive evaluation of these patients' condition by the clinicians, the fluid challenge was conducted. In it, at least $500 \mathrm{~mL}$ of crystalloid solution or at least $300 \mathrm{~mL}$ of colloidal solution was given within 30 minutes). An increase in $\mathrm{CO}$ of over $15 \%$ after fluid infusion was defined as volume responsiveness, and 43 patients were positive by both the NICOM built-in PLR test and the fluid challenge. When using $\Delta$ SVI to predict volume responsiveness of patients with circulatory shock (excluding cardiogenic shock), the AUC was 0.754 (95\% CI, 0.626-0.856), and the cut-off value was $18 \%$ (sensitivity: $88.37 \%$, specificity: $52.94 \%$ ), indicating that $\Delta$ SVI has value in predicting volume responsiveness of patients with circulatory shock (excluding cardiogenic shock).

\section{Discussion}

Non-invasive cardiac output monitoring is a hemodynamic monitoring technology based on biological response. In the past, some key technical problems such as low signalto-noise ratio of impedance and improper parameter calculation methods will lead to the accuracy of noninvasive hemodynamic measurement parameters. Inferior, but the biological response technology is not affected by the patient's po sition changes, environmental changes, humidity, electrode position and other factors. It is through the change of the phase shift of the radio frequency wave when the blood pumped from the ventricle passes through the chest cavity, thereby realizing the heart for output monitoring, Cardiac output (CO) is calculated as $\mathrm{CO}=\mathrm{SV} \times \mathrm{HR}$. The calculation of stroke volume $(\mathrm{SV})$ is: $\mathrm{SV}=\mathrm{C} \times \mathrm{VET} \times \mathrm{d} \Phi / \mathrm{dtmax}$. $\mathrm{C}$ is the constant of proportionality (it has been optimized considering the patient's age, gender and body type), and VET is the ventricular ejection time (determined by NICOM and ECG signals). And because noninvasive cardiac output monitoring is convenient and noninvasive, more and more attention has been paid. How consistent are the CO measurements from NICOM, PiCCO, and ultrasound? Does the NICOM built-in PLR test accurately predict volume responsiveness in critically ill patients?

Keren et al. performed continuous CO monitoring in 27 ICU patients after heart valve replacement surgery or coronary artery bypass graft surgery using SG catheters and NICOM, finding that NICOM was feasible to monitor CO in critically ill patients after cardiac surgery (8). However, the study of Rali et al. showed that for patients with cardiogenic shock, NICOM and thermodilution had poor consistency between their $\mathrm{CO}$ measurements. The reason for the poor agreement is that the bioelectrical impedance technique relies on the alternating current passing through the thoracic cavity, and cardiogenic shock is often accompanied by pulmonary edema, so the $\mathrm{CO}$ may be affected by the pulmonary edema and pulmonary interstitial edema (9). An analysis of the eight patients who fell outside the $95 \%$ CI in this study showed that all eight patients required a ventilator, five patients were complicated with severe pneumonia, and three patients were complicated with acute respiratory distress syndrome (ARDS). These patients had more pulmonary exudate and might have high right ventricular afterload, which may affect the consistency of the monitored CO. A 2011 study showed that the use of NICOM to monitor CO had limited value, and severe pulmonary arterial hypertension $(>60 \mathrm{mmHg})$, severe aortic valve insufficiency, and severe tricuspid valve insufficiency could cause it to overestimate CO, so NICOM was not validated in congenital heart disease patients with complex intracardiac shunts (10). In this study, the factors that may have interfered with the $\mathrm{CO}$ measured by NICOM were excluded during the patient screening, so their influence on the $\mathrm{CO}$ was minimized as far as possible.

The essence of shock is the lack of fluid, and the key to correcting shock is fluid resuscitation (11). When insufficient $\mathrm{CO}$ is suspected to be associated with volume, fluid resuscitation can be initiated. However, for critically ill patients, especially those with combined cardiac dysfunction or acute kidney injury, their circulatory fluid volume is small, and fluid overload is likely to occur, resulting in pulmonary edema, heart failure, and many other complications (12). Therefore, volume responsiveness should be assessed before fluid resuscitation. The tests for volume responsiveness include the 'classica' fluid challenge, the 'mini' fluid challenge, and the PLR test. The fluid challenge entails the infusion of at least 500 $\mathrm{mL}$ of crystalloid solution or at least $300 \mathrm{~mL}$ of colloidal solution within 30 minutes to observe whether the CO can be significantly increased (13). When carrying out the volume loading test, the faster the infusion rate, the smaller the required amount of liquid, and the smaller the differences between the effects of crystalloid solution and colloid solution (14). Toscani et al. also pointed out that the prolonged infusion could lead to decreased sensitivity of the 
volume responsiveness (15). In this study, the 'classic' fluid challenge was conducted. However, for different patients, the types and amounts of fluids used were inconsistent: both colloidal solution (human albumin) and crystalloid solution (compound sodium chloride solution and acetated Ringer's solution) were used. In addition, the infusion rate was not consistent, so we may have underestimated the number of patients who were positive in both the fluid challenge and NICOM built-in PLR test, thus affecting the experimental results, which was the biggest limitation of this study.

PLR is a reversible fluid challenge. It is simple to do and is not affected by spontaneous breathing and arrhythmia. It is considered an alternative to the classical volume loading test (16). However, the increases invenous return and cardiac preload caused by PLR is transitory, the maximum effect within 60 to 90 seconds, and the effect lasts approximately 10 minutes (17). Because the effect of PLR on $\mathrm{CO}$ is very short, hemodynamic monitoring equipment capable of real-time monitoring of $\mathrm{CO}$ should be used. In this study, the NICOM built-in PLR test was performed because it can monitor changes in $\mathrm{CO}$ in a timely manner. When performing PLR, the patient's Richmond AgitationSedation Scale (RASS) score should be kept within 0-2 points, and discomfort caused by postural changes should be minimized since pain and other discomfort can stimulate the sympathetic nervous system to raise CO. However, some patients cannot keep sedative during the test, resulting in the high CO detected by NICOM and yielding a falsepositive result of PLR.

A 2013 study used NICOM and PiCCO devices to compare the effects of PLR and fluid challenge on cardiac indices and found that the two devices were poorly correlated. Most of the patients included in that study also had ARDS, and the authors pointed out that the error in NICOM monitoring may have been related to lung injury and pleural effusion (18). For most patients in this study, their primary disease was not lung injury or pleural effusion. Among the 60 patients with $\Delta$ SVI $>10 \%, 17$ patients had $\Delta \mathrm{SVI}<10 \%$. Among these 17 patients, eight had ARDS or severe pneumonia, and one patient had a large amount of pleural effusion. However, considering the small sample, this study's results may not be reliable, so the reliability of using the NICOM built-in PLR test to predict volume responsiveness in patients with lung injury or pleural effusion needs to be further studied. Benomar et al. enrolled 75 patients after cardiac surgery and concluded that the bioreactance-based NICOM was clinically effective in predicting volume responsiveness from $\mathrm{CO}$ changes during the PLR test (19). In contrast, this study included not only patients after cardiac surgery but also other critically ill patients with various comorbidities in the Department of Critical Care Medicine. We also demonstrated the universality of using the $\Delta$ SVI measured by the NICOM built-in PLR test to evaluate volume responsiveness.

This study is a single-center study with a small sample size. Only one comparative evaluation was performed on critically ill patients who need to be monitored. The monitoring and comparison at multiple time points when the condition improves or worsens may increase the number of monitoring and evaluations for the same patient; The subjects of the study are mainly middle-aged and elderly patients with severely ill patients. The age group is relatively limited, and the severity of the condition is not combined with the APACHE II scoring system to make a quantitative evaluation of the severity of the disease. A large sample size is needed for comparative research.

In summary, NICOM can be an effective tool for hemodynamic monitoring and evaluation in critically ill patients. The $\triangle$ SVI obtained through the NICOM built-in PLR test can predict the volume responsiveness of patients with noncardiogenic circulatory shock to some extent. The accuracy of NICOM in the hemodynamic monitoring of critically ill patients still needs to be verified by large-scale clinical studies.

\section{Acknowledgments}

Funding: None.

\section{Footnote}

Reporting Checklist: The authors have completed the STARD reporting checklist. Available at http://dx.doi.org/10.21037/ apm-20-1731

Data Sharing Statement: Available at http://dx.doi. org/10.21037/apm-20-1731

Conflicts of Interest: All authors have completed the ICMJE uniform disclosure form (available at http://dx.doi. org/10.21037/apm-20-1731). The authors have no conflicts of interest to declare.

Etbical Statement: The authors are accountable for all aspects of the work in ensuring that questions related to the accuracy or integrity of any part of the work are 
appropriately investigated and resolved. This study followed medical ethics standards and was approved by the medical ethics committee of the hospital (approval number: 2019117). All procedures performed in this study involving huma $\mathrm{n}$ participants were in accordance with the Declaration of Helsinki (as revised in 2013). The patients or their family members were informed about the study protocols and signed informed consent forms.

Open Access Statement: This is an Open Access article distributed in accordance with the Creative Commons Attribution-NonCommercial-NoDerivs 4.0 International License (CC BY-NC-ND 4.0), which permits the noncommercial replication and distribution of the article with the strict proviso that no changes or edits are made and the original work is properly cited (including links to both the formal publication through the relevant DOI and the license). See: https://creativecommons.org/licenses/by-nc-nd/4.0/.

\section{References}

1. Perel A, Saugel B, Teboul J, et al. The effects of advanced monitoring on hemodynamic management in critically ill patients: a pre and post questionnaire study. J Clin Monit Comput 2016;30:511-8.

2. Ceccoin M, Backer D, Antonelli M, et al. Consensus on circulatory shock and hemodynamic monitoring. Task force of the European Society of Intensive Care Medicine. Intensive Care Med 2014;40:1795-815.

3. Jozwiak M, Monnet X, Teboul J. Less or more hemodynamic monitoring in critically ill patients. Current Opinion in Critical Care 2018;24:309-15.

4. Ayuela Azcárate JM, Clau-Terré F, Vicho Pereira R, et al. Consensus document on ultrasound training in Intensive Care Medicine. Care process, use of the technique and acquisition of professional skills. Med Intensiva 2014;38:33-40.

5. Raval NY, Squara P, Cleman M, et al. Multicenter evaluation of noninvasive cardiac output measurement by bioreactance technique. J Clin Monit Comput 2008;22: 113-9.

6. Squara P, Rotcajg D, Denjean D, et al. Comparison of monitoring performance of Bioreactance vs. pulse contour during lung recruitment maneuvers. Crit Care 2009;13: R125.

7. Min J J, Min J J, Lee J, et al. Utility of stroke volume variation measured using non-invasive bioreactance as a predictor of fluid responsiveness in the prone position.
Journal of Clinical Monitoring and Computing 2017;31: 397-405.

8. Keren H, Burkhoff D, Squara P. Evaluation of a noninvasive continuous cardiac output monitoring system based on thoracic bioreactance. Am J Physiol Heart Circ Physiol 2007;293:H583-9.

9. Rali AS, Buechler T, Van Gotten B, et al. Non-Invasive Cardiac Output Monitoring in Cardiogenic Shock: The NICOM Study. J Card Fail 2020;26:160-5.

10. García X, Mateu L, Maynar J, et al. Estimación del gasto cardíaco. Utilidad en la práctica clínica. Monitorización disponible invasiva y no invasiva. Medicina Intensiva 2011;35:552-61.

11. Huang HB, Xu B, Liu GY, et al. N-terminal pro-Btype natriuretic peptide for predicting fluid challenge in patients with septic shock. Ann Transl Med 2019;7:264.

12. Claure-Del Granado R, Mehta R L. Fluid overload in the ICU: evaluation and management. BMC Nephrol 2016;17:109.

13. Vincent J, Weil MH. Fluid challenge revisited. Crit Care Med 2006;34:1333-7.

14. Guinot PG, Bernard E, Defrancq F, et al. Mini-fluid challenge predicts fluid responsiveness during spontaneous breathing under spinal anesthesia: An observational study. Eur J Anaesthesiol 2015;32:645-9.

15. Toscani L, Aya H D, Antonakaki D, et al. What is the impact of the fluid challenge technique on diagnosis of fluid responsiveness? A systematic review and metaanalysis. Critical Care 2017;21:207.

16. Monnet X, Teboul JL. Passive leg raising: five rules, not a drop of fluid!. Crit Care 2015;19:18.

17. Mesquida J, Gruartmoner G, Ferrer R. Passive leg raising for assessment of volume responsiveness: a review. Curr Opin Crit Care 2017;23:237-43.

18. Kupersztych-Hagege E, Teboul JL, Artigas A, et al. Bioreactance is not reliable for estimating cardiac output and the effects of passive leg raising in critically ill patients. Br J Anaesth 2013;111:961-6.

19. Benomar B, Ouattara A, Estagnasie P, et al. Fluid responsiveness predicted by noninvasive Bioreactancebased passive leg raise test. Intensive Care Med 2010;36:1875-81.

Cite this article as: Zhu G, Zhang K, Fu Y, Hu Z. Accuracy assessment of noninvasive cardiac output monitoring in the hemodynamic monitoring in critically ill patients. Ann Palliat Med 2020;9(5):3506-3512. doi: 10.21037/apm-20-1731 